\title{
Model Development for Robust Optimal Control of Building HVAC
}

\author{
Saman Mostafavi ${ }^{1}$, Benjamin Futrell ${ }^{1}$, Robert W. Cox ${ }^{1}$ \\ ${ }^{1}$ UNC Charlotte, Charlotte, North Carolina
}

\begin{abstract}
Many control schemes have been proposed for improving the operation of variable-air volume HVAC systems. These approaches typically employ some form of simplified state-space model to predict the temperature evolution in each zone. One real-world challenge is the difficulty of developing such models with sufficient accuracy. Recent publications have shown that advanced controllers can utilize computationally efficient black-box models for control, but that such models must be developed using months or even years of data. The authors propose the following alternative process for model development: (1) begin with an EnergyPlus model developed during design; (2) calibrate that model using an approach such as the one demonstrated in Mostafavi (2017); (3) develop a surrogate model that runs hundreds of times faster than the EnergyPlus model; (4) utilize the surrogate model to develop the controller without having to acquire extensive historical data. This approach allows a controller to be developed in a matter of days rather than months or years. Critical to the process is a calibrated EnergyPlus model that accurately reflects both energy consumption and thermal behavior.
\end{abstract}

\section{Introduction}

Buildings account for approximately $40 \%$ of the primary energy consumed in the United States each year DOE (2017). Data suggests that faulty or poorly operated systems are responsible for approximately $20 \%$ of this consumption Katipamula and Brambley (2005). Improved building energy efficiency can directly result in significant reduction of energy consumption, carbon emission, and utility expenses. Among the efforts to address this issue, advanced building control has shown efficiency improvements through better coordination of various HVAC components, adaption to environmental changes and elimination of human errors. Current building controls are typically based on a decentralized architecture. For example, the air handling unit (AHU) has its own control mission to satisfy supply air temperature set-point and variable air volume (VAV) units are controlled to meet their flow set-points to maintain zone temperature. Advanced building controls such as optimization-based control, however, tends to bridge the subsystem operation together and leverage the trade-offs between HVAC components (Brambley et al. (2005); Katipamula et al. (2012)). Past studies have indicated a gap between optimal and current building operational schedules. It has been suggested that, optimal control of HVAC alone can result in a reduction in whole building energy consumption by up to $10 \%$ Roth et al. (2005). This can be achieved by continuously adjusting set-points and/or ventilation to minimize energy cost while maintaining comfort.

This can be formulated as an optimal control task, deriving a predictive model from historical building data (e.g. occupancy and load patterns, set points etc.) and weather information to use an optimization task. The problem however, is complex, multivariate and potentially non-convex depending on the model definition and it's constraints. Several studies have suggested using Model Predictive Control (MPC) in solving the aforementioned problem as MPC provides a systematic implementation option using a system model and an optimization algorithm to adjust the control set-points dynamically while automatically satisfying steady state and dynamic component and operation constraints Bengea et al. (2014).

MPC (Also know as receding horizon control) refers to a class of control algorithms that utilize a model of a system and forecasts to predict and optimize the systems future response Morari and Lee (1999). After selecting the optimal control inputs for a minimum cost function, the first control sequence is applied. This process is then repeated for the next time steps, each time only utilizing one input. For example, the controller can be set to minimize energy use over a period. The models are trained using historical data. Ideally, the model should be adaptive (or else refined) over time to deal with changes in the environment. MPC for supervisory control of buildings has been proposed by Kelly (1988) and has been studied with a number of experimental results including optimizing building heating systems (Širokỳ et al. (2011)) and multi-objective optimization scheme for commercial offices (West et al. (2014). Recently simulation and small-scale test-bed experiments have been reported illustrating potential benefits (e.g., Henze et al. (2004); Clarke et al. (2002); Li et al. (2014)). 
One of the more comprehensive studies in optimization of conventional HVAC systems in a full-scale building has been carried out by Bengea et al. (2014) which utilizes the communication of the optimal control scheme with the building automation system, the controlled set-points and the component-level feedback loops to optimize the measured energy and indoor comfort. In the latest efforts, there has been an interest in applying Deep-Reinforcement Learning (DRL) for building HVAC control (Wei et al. (2017)).

Although MPC can provide significant HVAC energy savings, there are practical difficulties. Perhaps the most challenging issue is the need to develop dynamic models possessing sufficient accuracy for use in control. Developing such models requires extensive field data collection over a range of different operating conditions. Most previous work demonstrating the energy-saving benefits of MPC have been limited to short time periods lasting only several days, and a major reason for this is the difficulty of constructing accurate models Bengea et al. (2014). As shown in the Approach section below, a model developed over a certain set of conditions may or may not accurately predict behaviors during other conditions.

This paper proposes to address the modeldevelopment problem by creating a calibrated white-box model that can effectively serve as a "digital twin" for the real building. This white-box model, which is not practical for control, can be used offline to generate a large training set reflective of the many conditions a building is likely to experience. This training set is then used to produce a computationally efficient, black-box model used as a surrogate for real control. The authors propose the following specific approach: (1) begin with an EnergyPlus model developed during design; (2) calibrate that model using an approach such as the one demonstrated in Mostafavi (2017); (3) develop a surrogate model that runs hundreds of times faster than the EnergyPlus model; (4) utilize the surrogate model to develop the controller without having to acquire extensive historical data. The surrogate model developed here is an adaptive black-box model developed using the Extreme Gradient Boosting (XGB) algorithm proposed by Chen and Guestrin (2016), a popular Machine Learning (ML) choice in time-series modeling. Recall that the value of this approach lies in the development of a calibrated white-box model that can efficiently generate a large training set.

The remaining sections of this paper describe the proposed optimal control procedure. First, we discuss the underlying theory motivating the decision to develop a black-box model rather than a linear statespace model. We then describe the specific details of our approach, and finally compare our approach's ability to improve the optimal control task. This com- parison is performed using the US Department of Energy Medium Office model DOE (2017), and the primary output of the optimal control system is the supply air temperature setpoint. Our approach is shown to reduce the HVAC electrical energy consumption while maintaining comfort in the zones. The paper concludes with a discussion on next steps.

\section{Motivation}

For many years MPC has been widely utilized in industry as an effective means to deal with multivariate constrained control problems. Morari and Lee (1999) point out that model development is by far the most critical and time-consuming step in implementing a model predictive controller. It is estimated that, in a typical commissioning project, modeling efforts can take up to $90 \%$ of the cost and time. Arguably, the most expensive aspect for developing a model is often the availability of data. Today, more and more Building Management Systems (BMS) are being installed and retrofit. Even when such systems are available, it can take a long time to acquire a data set sufficient for proper model identification. This section explores this question utilizing a motivating example.

\section{The Model Identification Process}

There are four general challenges in the modelidentification process (Ljung (1998)):

- Model structure determination

- Test input signal design

- Identification algorithm

- Model validation

The purpose of the test input signal is to inject excitation signals into the system and collect the resulting output signals, which reveal the key dynamical properties of the system. Ljung (1998) shows that a Pseudo Random Binary Sequence (PRBS) would provide the necessary excitation. Model structure determination is by far the most difficult and pressing issue Morari and Lee (1999). Typically, an inputoutput model of the following form is identified:

$$
y(k)=F(\phi(k), \theta)+\epsilon(k)
$$

where $\phi$ is the regressor vector containing the delayed input and output terms and $\theta$ is a vector containing the unknown parameters. When applying MPC to control building HVAC systems, most researchers utilize a linear state space model of the form Li et al. (2012), Bengea et al. (2014)

$$
\begin{array}{r}
x_{k+1}=A x(k)+B u(k)+w(k) \\
T_{z}(k)=C x(k)+D u(k)
\end{array}
$$

where $k$ is the current time step, $x$ is the state vector, $u(k)$ is the input vector, $T_{z}$ is the zone temperature and $A, B, C, D$ are system matrices which are estimated using data for each zone of the building. 


\section{Example: The Challenge in Creating an Ac- curate State-Space Model}

To demonstrate the difficulty in developing an effective state-space model, the authors developed one for the Department of Energy's medium-sized commercial office building reference model and examined its predictive capabilities under different conditions. Table 1 lists the parameters for the example building DOE (2017). The state-space model takes the form given in Eq. 2. Each zone has its own model, and thus the output of each is denoted as $T_{z i}$. Table 2 lists the variables in the input vector $u$. These variables were determined after an intense grid-search process for finding the optimal set that results in best training of the model. As suggested by Li et al. (2014), a Kalman filter is used for prediction of initial states $\hat{x}_{0}$ that would result in a more accurate estimation of zone temperature prior to predictions.

Table 1: Key building attributes in the medium-sized office reference model.

\begin{tabular}{|l|l|}
\hline Building Attribute & Value \\
\hline Gross Floor Area & $1661 \mathrm{~m}^{2}$ \\
Wall U-value & $0.70 \mathrm{~W} / \mathrm{m}^{2}-\mathrm{K}$ \\
Window U-value & $4.60 \mathrm{~W} / \mathrm{m}^{2}-\mathrm{K}$ \\
Glass SHGC & 0.25 \\
Cooling Thermostat Set Point & $24.0{ }^{\circ} \mathrm{C}$ with $26.7{ }^{\circ} \mathrm{C}$ \\
& unoccupied setback \\
Heating Thermostat Set Point & $21.0{ }^{\circ} \mathrm{C}$ with $15.6{ }^{\circ} \mathrm{C}$ \\
Lighting Power Density & unoccupied setback \\
Equipment Power Density & $10.8 \mathrm{~W} / \mathrm{m}^{2}$ \\
Ventilation Rate & $8.07 \mathrm{~W} / \mathrm{m}^{2}$ \\
Infiltration Rate & $000431773 \mathrm{~m}^{3} / \mathrm{s}-\mathrm{m}^{2}$ \\
Occupancy Density & $0.10 \mathrm{ACH}$ occupied \\
Occupied Operational & $18.58 \mathrm{~m} 2 /$ people \\
Schedule & $6: 00$ AM to $10: 00$ PM \\
& Mon. through Fri. \\
HVAC System & $7: 00$ AM to $5: 00$ PM Sat. \\
& Unoccupied Sun. \\
& DX roof top units \\
serving VAV terminals \\
units with electric reheat \\
\hline
\end{tabular}

To identify the model parameters in the $A, B, C$ and $D$ matricies for each zone, one must utilize a set of inputs that is persistently exciting. Ideally, the input signal would have a frequency spectrum that is

Table 2: Input variables used in the state-space model in Eq. 2.

\begin{tabular}{|l|l|}
\hline Variable & Description \\
\hline$T_{\text {sa }}$ & Supply air temperature \\
$C_{\text {set }}$ & Cooling setpoint \\
$H_{\text {set }}$ & Heating setpoint \\
$\dot{\mathrm{m}}_{\mathrm{sa}}$ & Supply air flow rate \\
$T_{\text {dew }}$ & Dew point of outside air \\
$T_{\text {oat }}$ & Outside air temperature \\
$H$ & Humidity of outside air \\
Occ & Building Occupancy \\
\hline
\end{tabular}

nearly constant across all frequencies of interest. The only control input varied in this case is the supply air temperature setpoint, and it should be adjusted over an appropriate, bounded range. A pseudo random control sequence is generated utilizing the following equation adapted from the Wiener process

$$
T_{s a}[k]=T_{s a}[k-1]+i \times N\left(j, \delta^{2} d t ; k-1, k\right)
$$

where $N(a, b ; t 0, t 1)$ is a normally distributed random variable with mean $a$ and variance $b ; \delta$ and $d t$ are Wiener process parameters that determine the time step and the speed of the random walk respectively; and $i \in\{-1,1\}$ and $j$ are reset parameters controlling the boundary and size of the jumps ( $i$ reverses the direction of the process every time an upper/lower bound is passed). Figure 1 demonstrates an instance of such a sequence.

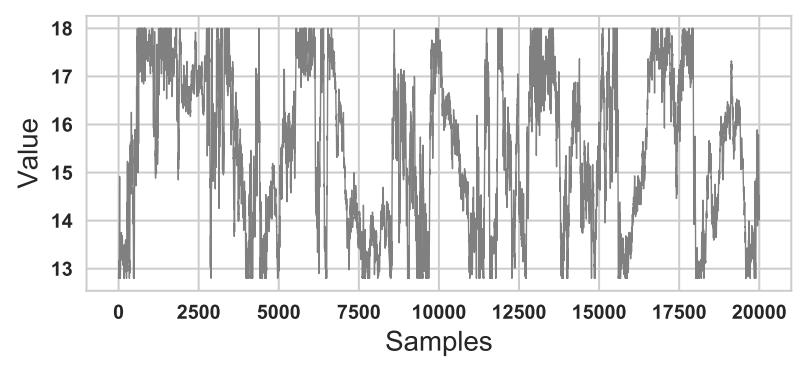

Figure 1: Example of a bounded pseudo random sequence for $T_{\text {sa }}$, assuming boundaries at 12.8 degrees $C$ and 18 degrees $C$.

Model parameters were identified during a one-week training period during the first week of March. The use of one week of training data is consistent with the literature ( $\mathrm{Li}$ et al. (2014) and Bengea et al. (2014)). Given that such a small training set necessarily includes only a portion of possible weather conditions, some inputs are not sufficiently excited. Figure 2 demonstrates the impact of this problem. Both images in that figure show a histogram of residuals between the predicted and simulated temperature in a given zone over two different time periods. The top graph shows the residuals during the training period in the first week of March; the bottom graph shows the residuals in the last week of March, when the weather was colder. Even though the two periods are only a few weeks apart, it is clear that the model must be adapted to address the change in weather in late March.

Finally, let us consider the effects of the predictive capabilities of the model on optimal control problem. The optimal control problem is defined as:

$$
V(x)=\max _{u} E_{e}[R[x, u]+V(f(x, u, e))] .
$$




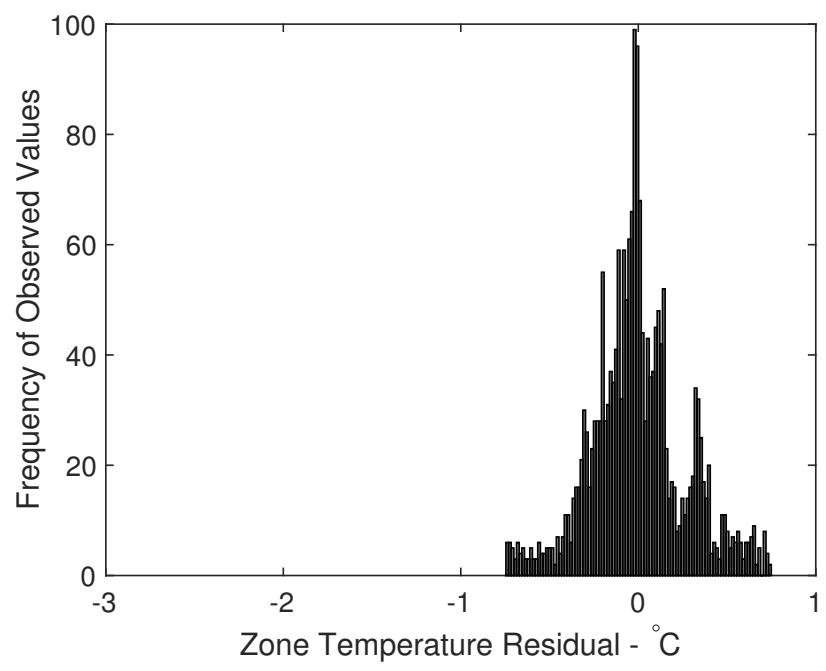

(a)

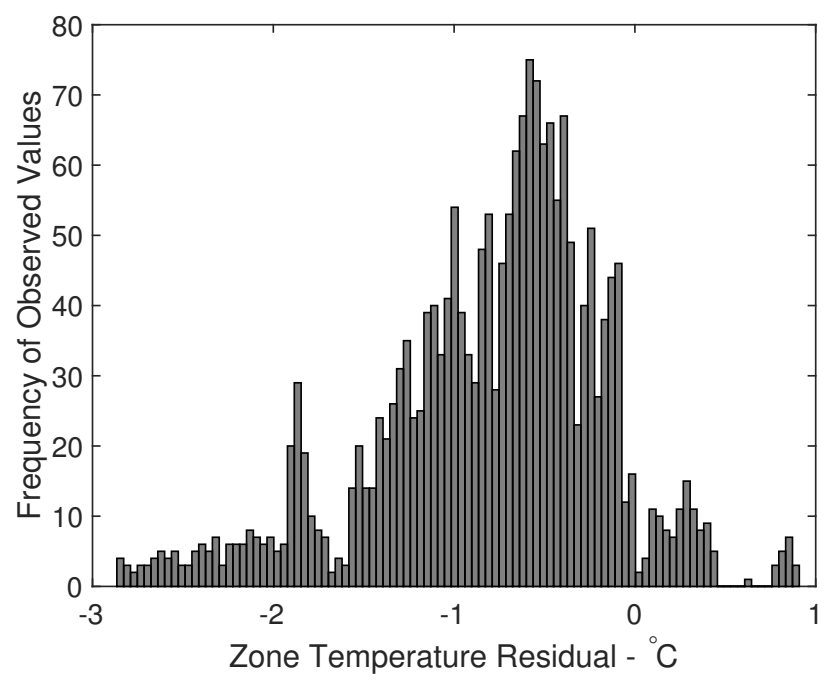

(b)

Figure 2: Zone temperature residuals for the statespace model for a given zone in the reference model. (a) Residuals from the training period in the first week of March. (b) Residuals during the last week of March. Data is recorded every 3 minutes.

Where $V(x)$ is defined to be the value obtained from solving the optimal control problem with initial condition $x$, input signal $u$ and error term $e$ for statetransition map, $f$ ( $E$ is the expected value operator). This equation, known as Bellmans equation defines a powerful recursive formula for $V$ but is very costly to solve. The receding horizon control approach to solving the problem is defined as:

$$
\begin{aligned}
& \max _{u_{t}} E_{e_{t}}\left[\sum_{t=0}^{N} R\left[x_{t}, u_{t}\right]+V\left(x_{N+1}\right)\right] \\
& \text { subject to } x_{t+1}=f\left(x_{t}, u_{t}, e_{t}\right) \\
& \left(x_{0} \text { given }\right) .
\end{aligned}
$$

Here the cost is unrolled beyond one step given that the cost-to-go $N$ steps in the future is calculated. Though this is trivial, it is very important to note that the longer the time horizon, the less one has to worry about the value function $V$ being accurate. A prerequisite to this is to have available predictions for the window. It is common practice to solve MPC for 2 to 3 hour window (Bengea et al. (2014); Li et al. (2014)). Li et al. (2012) points the shortcomings of the trained state-space model for longer windows up to 8 hours. In the result section, we show that having an accurate prediction window would improve the results of optimal control.

\section{Approach}

Previous sections have described the basic procedure used to do optimal control in building. Here, we start from a calibrated building model generating data replicating the real building thermal response under different weather and load conditions. The model is designed in EnergyPlus and therfor is computationally very heavy and not suitable to fast MPC. Using the data generated from EnergyPlus, we fit a surrogate model that captures the physics based model behaviour with high accuracy. Our model considers weather information, HVAC supply air temperature, thermostat set-points and operational schedules to estimate zone temperatures and energy consumption. The control objective is to reduce HVAC energy consumption while maintaining(and/or improving) thermal comfort. The controlled variable is supply air temperature set-point. The impact of the prediction horizon length on the optimality of the controller is explored more deeply in the next section. The remainder of this section describes the details of the surrogate modeling and the optimal control process.

\section{Surrogate Model}

Consider the model described in equation 1 . The task of training the model amounts to finding the best parameters $\theta$ that best fit the training data $\phi(k)$ and $y(k)$. In order to train the model, we define an objective function to minimize the discrepancy between the outputs of $F$ and $y(k)$. This is described as:

$$
\operatorname{obj}(\theta)=L(\theta)+\Omega(\theta)
$$

where $L(\theta)$ is the training loss function, often defined for regression tasks as a mean square error (MSE):

$$
L(\theta)=\sum_{i}\left(y_{i}-\hat{y}_{i}\right)^{2}
$$

and $\Omega(\theta)$ is the regularization term added to avoid over-fitting to training data (In other words, encouraging simpler models). It is in their choice of models for minimizing the defined objective function in equation 6 that Machine Learning algorithms differ from 
one another. One particular success story in time series regression has been Extreme Grading Boosting (XGBoost) Chen and Guestrin (2016). A member of boosting algorithms family, a set of weak learners, in this case CART trees, is combined into a weighted sum that represents the final output of the boosted regressor. Considering MSE as loss function, equation 6 is derived as:

$$
\begin{array}{r}
\operatorname{obj}^{(t)}=\sum_{i=1}^{n}\left[2\left(\hat{y}_{i}^{(t-1)}-y_{i}\right) f_{t}\left(x_{i}\right)+f_{t}\left(x_{i}\right)^{2}\right]+\Omega\left(f_{t}\right) \\
\text { where, } \hat{y}_{i}=\sum_{k=1}^{K} f_{k}\left(x_{i}\right), f_{k} \in \mathcal{F}
\end{array}
$$

where $K$ is the number of trees, $f$ is a function in the functional space $\mathcal{F}$, and $\mathcal{F}$ is the set of all possible CARTs (Rätsch et al. (2001)). By defining the optimization problem, i.e. minimizzing equation 8 , we can now train the boosted tree model. The described process is the general approach in learning adaptive boosted tree models for a regression task. XG Boost is a special case of this family motivated by the formal principle introduced and is developed with both deep consideration in terms of systems optimization and principles in machine learning. Further details about the algorithm can be found in Li et al. (2014).

\section{Optimal Control Problem}

We implement the controller using MPC procedure described in the previous section where an optimization problem over $\mathrm{N}$ time steps (the control and prediction horizon) is solved at each step. The output of the algorithm is an optimal input for the chosen window. At each iteration, we only take the first step for the control and roll the window one step and repeat the process. The controller objective is minimizing the electrical energy of HVAC, constituted of cooling coil, heating coil and fan energy, while maintaining or improving comfort. By that we mean the zone thermostat dead-band should be kept at all times and if possible narrowed. To reduce the wear and tear of the air conditioning unit, we add a penalizing term to discourage frequent changes in the supply air temperature. The optimization problem is then formulated as:

$$
\max _{T_{s a}}\left[\alpha \sum_{k=1}^{N} \dot{Q}_{H V A C}+R E G\right]
$$

subject to $T_{s a} \in\left[T_{0}, T_{1}\right]$

$\left(x_{0}\right.$ given $)$.

where $N$ is the window size for optimization and REG is the regularization term that should be minimized along with $Q$, the HVAC energy. Regularization term is added to ensure faster convergence and also for two other main reasons: One is to narrow the thermostat dead-band using a slack variable and the other is to avoid frequent change in the supply air temperature. This term is therefore defined as:

$$
\begin{array}{r}
R E G=\beta \sum_{k=1}^{N}\|g\| \\
+\gamma \sum_{k=1}^{N}\left\|T_{s a}^{k}-T_{s a}^{k-1}\right\|
\end{array}
$$

here $\alpha, \beta$ and $\gamma$ are tuning parameters, chosen to damp or encourage large steps in each direction as needed. $g$ ensures that $T_{z}-T_{z}^{C s e t}<s_{k}, T_{z}-T_{z}^{H \text { set }}>$ $s_{k}$ for some margin $s_{k}$, improving the dead-band in the process.

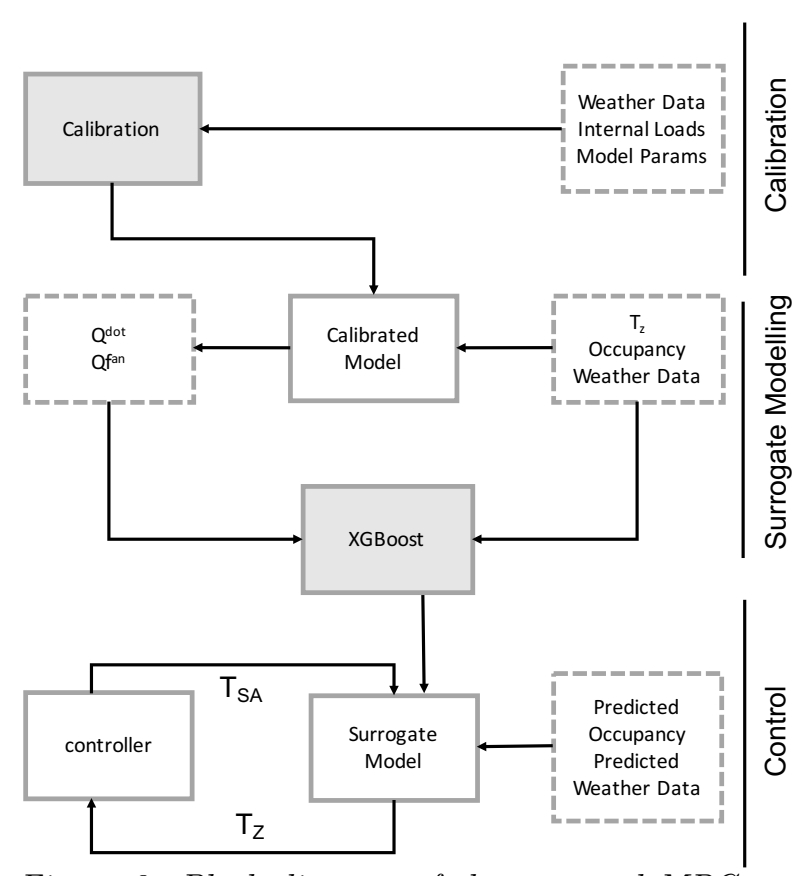

Figure 3: Block diagram of the proposed MPC controller.

\section{Simulation Study: Demonstrating the Importance of Calibrated Model}

To explore the impact of using a surrogate model in the optimal control process, the authors used the medium-sized office reference model described previously (DOE (2017)). The authors performed model predictive control using two approaches.

1. Method 1 - Model Predictive Control using State-Space Model: A second order state space model is developed using the approach described in the motivation section as in Li et al. (2014)

2. Method 2 - Model Predictive Control using proposed surrogate model: A surrogate model is trained using data generated from the 
calibrated EnergyPlus model with Extreme Gradient Boosting as is proposed in this paper.

To train the surrogate model, the authors have considered doing a sensitivity analysis first. Another point of consideration was the availability of data for predictive purposes. Considering these two bounding criteria, a subset as describe in 3 was considered for training. It is safe to assume that accurate weather prediction is available today that can be acquired either free or with relatively low cost from online services. This feature seems to be supported by and large by the state of the art BMS. Occupancy patterns can be estimated from historical data. As in previous section, to ensure proper excitation, Supply air temperature is generated using the pseudo random sequence method. These inputs are then trained to learn separate models for each zone temperature and the HVAC loads, i.e., cooling, heating and fan energy.

Table 3: Input and output variables used for training in the training process of XGBoost.

\begin{tabular}{|l|l|}
\hline Variable & Description \\
\hline$T_{z i}$ & Temperature in zone $i$ \\
$T_{s a}$ & Supply air temperature $i$ \\
Solar $_{\text {rad }}$ & Solar radiation \\
Azim & Azimuth \\
$\dot{\mathrm{m}}_{\text {sa }}$ & Supply air flow rate \\
$T_{\text {dew }}$ & Dew point \\
$T_{\text {oat }}$ & Outside air temperature \\
$H$ & Humidity \\
Occ & Occupancy \\
$\dot{\mathrm{Q}}_{\text {cool }}$ & Cooling coil energy \\
$\dot{\mathrm{Q}}_{\text {heat }}$ & Heating coil energy \\
$\dot{\mathrm{Q}}_{\text {fan }}$ & Fan energy \\
\hline
\end{tabular}

It is important to note that in Boosting algorithms, there are many hyper parameters that can effect the learning process and would need proper tuning. These include type of the tree model, booster parameters such as depth and number of trees, and learning rate and regularization which guide the overall optimization task described before. To tune these parameters, the authors ran a grid search process using 5 fold cross-validation to avoid over-fitting. To make results reproducible, the model hyper-parameters are presented in Table 4 but the readers are encouraged to do the same process for best results for other applications.

Figure 4 demonstrates the error percentage between the EnergyPlus outputs and the trained surrogate models. Figures a-d indicate training phase and the rest shows the validation. Training was done using 3 month of minute based data and 5 fold crossvalidation and validation was carried out for 6 month of unobserved data. (a) and (e) shows temperature error for the main zone. ; (b) and (f) show error of cooling coil power; (c) and (g) show error of heating
Table 4: Hyper-parameters used in training XGBoost Model.

\begin{tabular}{|l|l|}
\hline Parameter & Value \\
\hline Booster & gbtree \\
Colsample by tree & 0.8 \\
Learning Rate & 0.05 \\
number of estimators & 1000 \\
reg $_{\alpha}$, reg & 0,1 \\
max depth of tree & 15 \\
\hline
\end{tabular}

coil power; and (d) and (h) show error of fan energy. Although it might not look very promising at first glance, we would like to emphasize that this is purely due to the number of points plotted in each figure (more than 30000), and the red line indicating the $95 \%$ confidence interval of error together with histograms on the side for each figure indicate a very narrow band of error of less than $5 \%$ with minimal positive or negative bias. Note that during the validation period, weather has changed significantly but the surrogate model is still able to do accurate prediction of the objectives of the learning process.

Figure 5 demonstrates the outcome of model predictive control for a day in March. In here, we have compared the two different aforementioned methodologies for model training and have utilized each for solving the receding horizon window problem. Our proposed methodology is able to reduce the coil energy significantly with an increase in fan energy. This comes from the fact that an increase in the supply air temperature would decrease the cooling coil energy but demands a higher flow for keeping the set points. Overall, it can be said that the energy consumption is reduced considerably. The bottom figure shows an improvement in the zone temperature as the proposed approach is able to keep the set-point $1^{\circ} \mathrm{C}$ below the cooling set-points at all times. Upon further analysis, it was discovered that an under estimation of the zone temperature in the state-space model was resulting in the model predictive control under performing as the model could not anticipate the passing of the cooling set-point, resulting in unnecessary high supply air temperature which in return increase the electrical consumption of the HVAC. In short, the proposed approach outperforms the traditional in both reducing the energy consumption and narrowing the dead band (objectives of the optimization problem).

Figure 6 demonstrates the comparison between the performance of the two approaches in three different cases:

- Case1: State-Space model is trained and used for MPC with a gap of 7 days. Control day max outside air temperature is $22^{\circ} \mathrm{C}$.

- Case2: State-Space model is trained and used for MPC with a gap of 3 weeks. Control day max outside air temperature is $19^{\circ} \mathrm{C}$. 


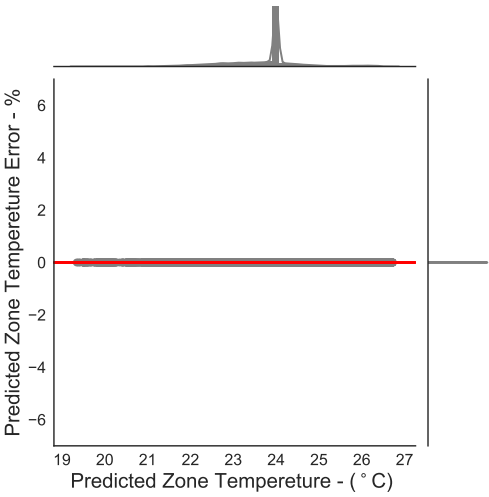

(a)

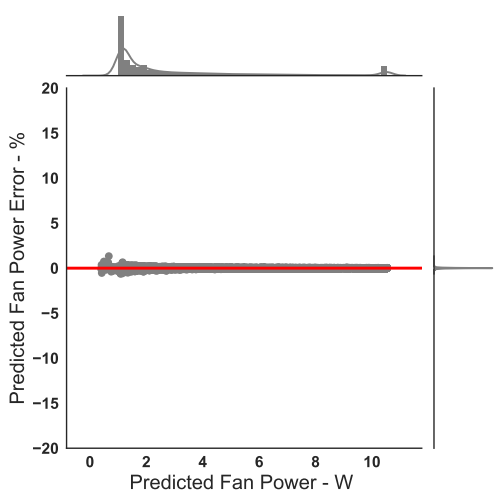

(d)

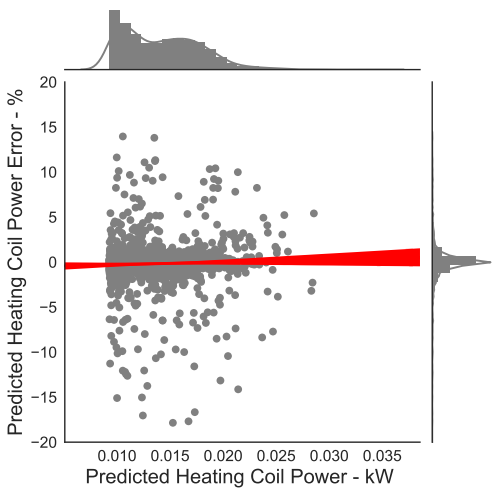

(g)

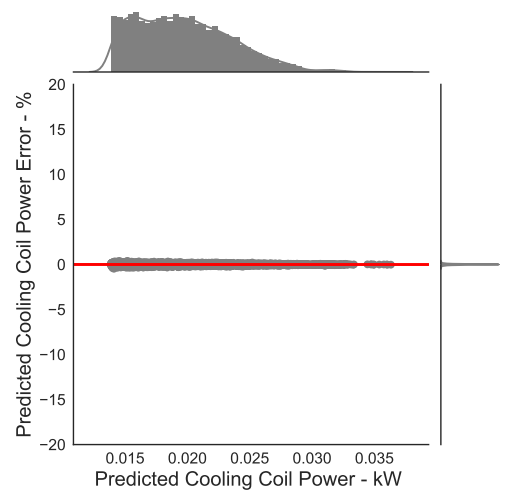

(b)

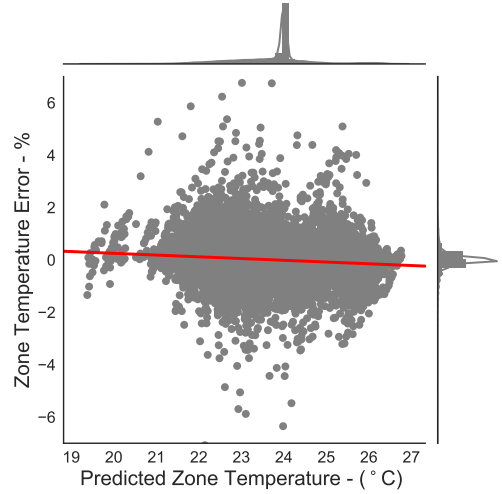

(e)

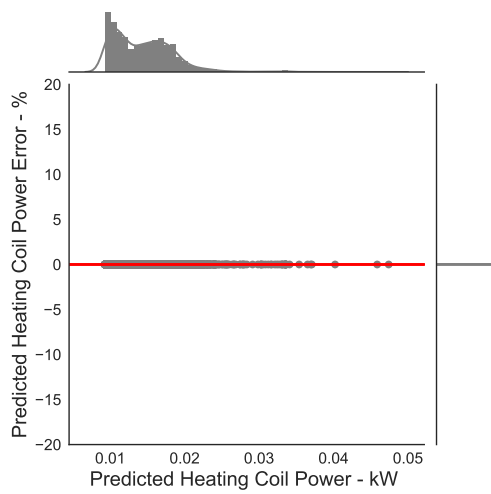

(c)

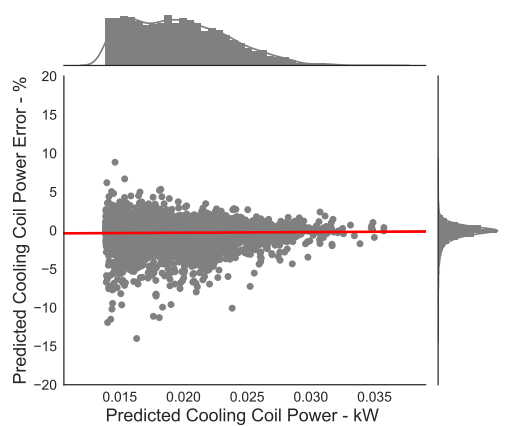

(f)

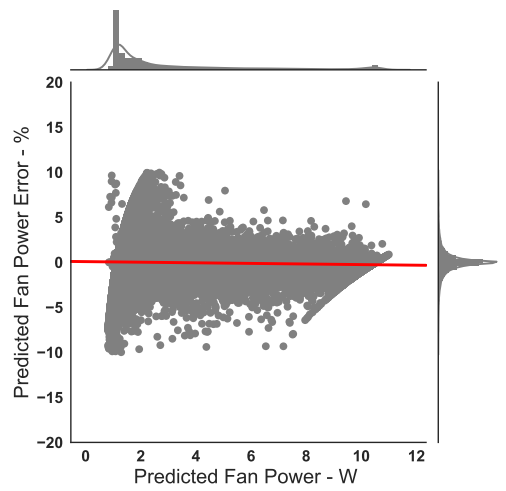

(h)

Figure 4: Comparison of Error between the EnergyPlus outputs and the trained surrogate model. Figures a-d indicate training phase and the rest shows the validation. Training was done using 3 month of minute based data and 5 fold cross-validation and validation was carried out for 6 month of unobserved data. (a) and (e) shows temperature error for the main zone. ; (b) and ( $f$ ) show error of cooling coil power; (c) and (g) show error of heating coil power; and (d) and (h) show error of fan power. The red line indicates the $95 \%$ confidence interval of error for each figure. Note that during the validation period, weather has changed significantly but the surrogate model is still able to do accurate prediction within ranges required for the MPC task.

- Case3: State-Space model is trained and used for MPC with a gap of 1 day. Control day max outside air temperature is $24^{\circ} \mathrm{C}$.

Note that for MPC2 all of the control days are unobserved periods outside of the training phase. The varying performance in 3 graphs for MPC2 is due to the fact that control is carried out in three different days. As discussed before, the predictive performance of the state-space model is worse as the window between training and control day increases. In this case, if trained a day before, state-space model can produce desirable zone temperature predictions but a gap of a few weeks can significantly effect that. Our proposed approach managed to outperform the traditional ap- 
proach without the need to re-calibrate the model.

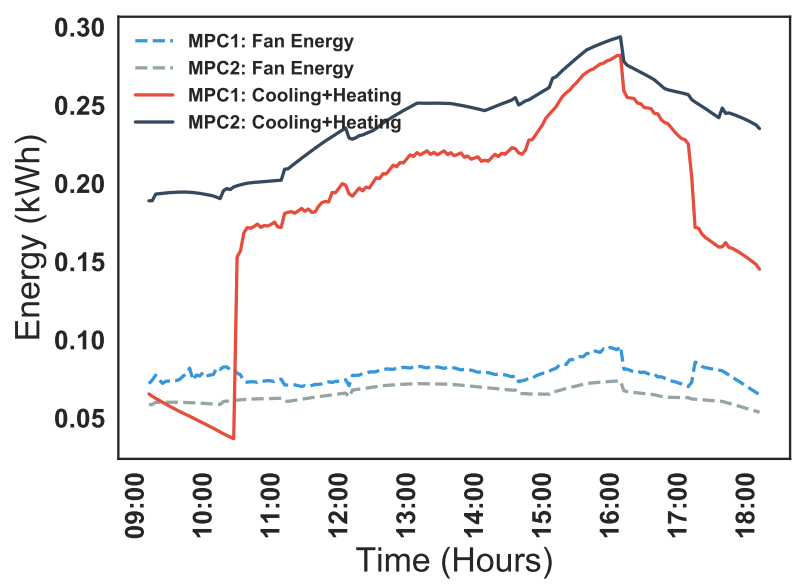

(a)

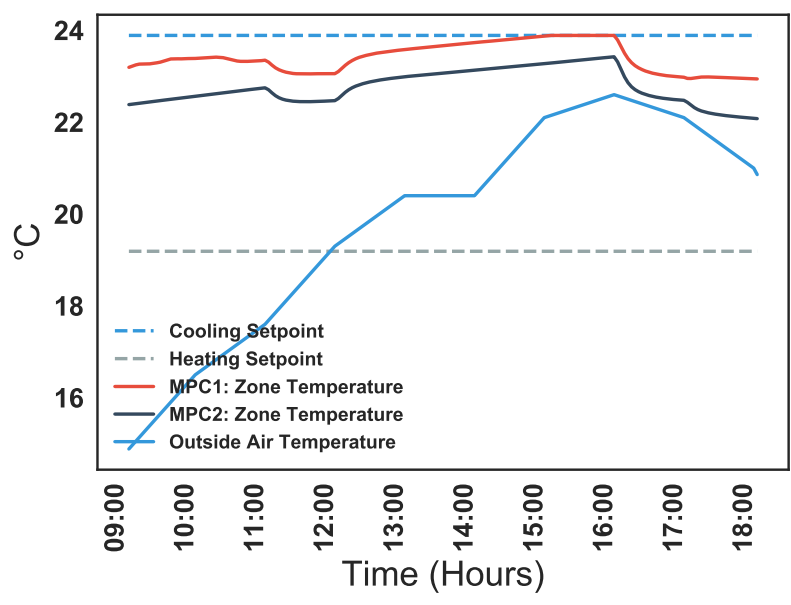

(b)

Figure 5: outcome of model predictive control for a day in March. (a) Total cooling, heating and fan energy compared between the traditional and proposed MPC approach. (b) Comparison of zone temperatures with regards to set-points and outside air temperature. The proposed approach outperforms the traditional in both reducing the energy consumption and narrowing the dead band (objectives of optimization).

\section{Conclusion}

This paper has demonstrated an approach for improving the models used for optimal control of building HVAC. The key element is the existence of an accurate white-box model in a tool such as EnergyPlus that can be used as a "digital twin." With such a model, one can generate a large training set containing sufficient variations in all key variables driving energy consumption. Note, however, that this paper has simply shown that a surrogate model for control can be developed using an EnergyPlus model. In practice, one would first need to calibrate such a white-box model. The authors have previously demonstrated an approach for such a calibration in Mostafavi et al.

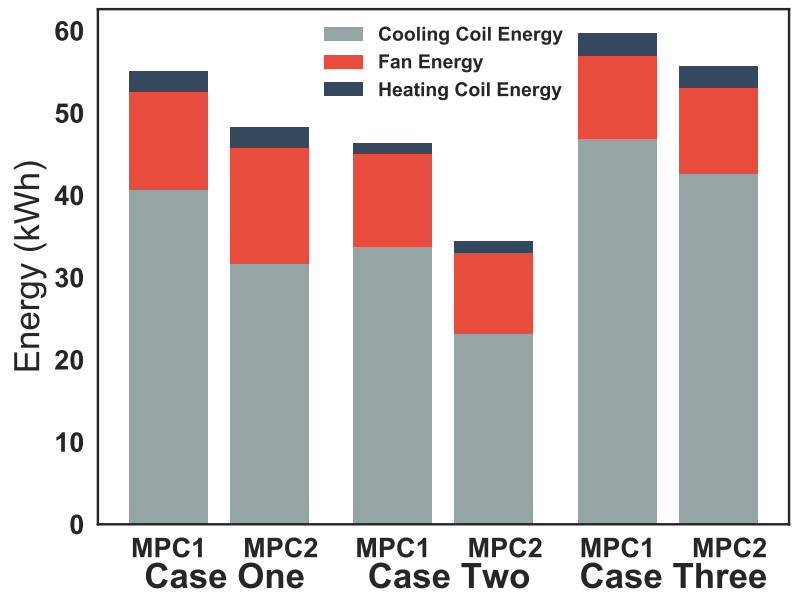

Figure 6: a comparison between the performance of the two approach in three different cases: Case1: State-Space model is trained and used for MPC1 with a gap of 7 days. Control day max outside air temperature is $22^{\circ} \mathrm{C}$; Case2: State-Space model is trained and used for MPC1 with a gap of 3 weeks. Control day max outside air temperature is $19^{\circ} \mathrm{C}$; and Case3: State-Space model is trained and used for MPC1 with a gap of 1 day. Control day max outside air temperature is $24^{\circ} \mathrm{C}$. The proposed surrogate model is used for MPC2. All three cases are unobserved periods for the surrogate model.

(2017). Future work will examine the application in a real building.

\section{Acknowledgement}

This material is based upon work supported by the National Science Foundation under grant no. IIP1161031.

\section{References}

Bengea, S. C., A. D. Kelman, F. Borrelli, R. Taylor, and S. Narayanan (2014). Implementation of model predictive control for an hvac system in a mid-size commercial building. HVACER Research 20(1), 121-135.

EERE Publication and Product Library, Washington, DC (United States) (2005). Advanced sensors and controls for building applications: Market assessment and potential RED pathways.

Chen, T. and C. Guestrin (2016). Xgboost: A scalable tree boosting system. In Proceedings of the 22nd acm sigkdd international conference on knowledge discovery and data mining, pp. 785-794. ACM.

Clarke, J., J. Cockroft, S. Conner, J. Hand, N. Kelly, R. Moore, T. OBrien, and P. Strachan (2002). Simulation-assisted control in building energy management systems. Energy and buildings 34(9), 933940 . 
DOE (2017). Reference buildings by building type: Medium office.

Henze, G. P., D. E. Kalz, C. Felsmann, and G. Knabe (2004). Impact of forecasting accuracy on predictive optimal control of active and passive building thermal storage inventory. HVACER Research 10(2), 153-178.

Katipamula, S. and M. R. Brambley (2005). Methods for fault detection, diagnostics, and prognostics for building systemsa review, part I. HVACER Research 11(1), 3-25.

Pacific Northwest National Lab.(PNNL), Richland, WA (United States) (2012). Small-and mediumsized commercial building monitoring and controls needs: A scoping study.

Kelly, G. E. (1988). Control system simulation in north america. Energy and Buildings 10(3), 193202.

Li, P., M. Baric, S. Narayanan, and S. Yuan (2012). A simulation-based study of model predictive control in a medium-sized commercial building. In 2nd International High Performance Buildings Conference at Purdue, pp. 16-19.

Li, P., D. Li, D. Vrabie, S. Bengea, and S. Mijanovic (2014). Experimental demonstration of model predictive control in a medium-sized commercial building.

Ljung, L. (1998). System identification. In Signal analysis and prediction, pp. 163-173. Springer.

Morari, M. and J. H. Lee (1999). Model predictive control: past, present and future. Computers $\&$ Chemical Engineering 23(4-5), 667-682.

Mostafavi, S., B. Futrell, and R. W. Cox (2017). A calibration process focused on predicting both energy performance and indoor thermal conditions. In IBPSA Build. Simul. Conf, Volume 2017.

Rätsch, G., T. Onoda, and K.-R. Müller (2001). Soft margins for adaboost. Machine learning 42(3), $287-320$.

Roth, K. W., D. Westphalen, M. Y. Feng, P. Llana, and L. Quartararo (2005). Energy impact of commercial building controls and performance diagnostics: market characterization, energy impact of building faults and energy savings potential. Prepared by TAIX LLC for the US Department of Energy. November. 412pp (Table 2-1).

Širokỳ, J., F. Oldewurtel, J. Cigler, and S. Prívara (2011). Experimental analysis of model predictive control for an energy efficient building heating system. Applied energy 88(9), 3079-3087.
Wei, T., Y. Wang, and Q. Zhu (2017). Deep reinforcement learning for building hvac control. In Proceedings of the 54th Annual Design Automation Conference 2017, pp. 22. ACM.

West, S. R., J. K. Ward, and J. Wall (2014). Trial results from a model predictive control and optimisation system for commercial building hvac. Energy and Buildings 72, 271-279. 